\title{
Status of the windowless gaseous tritium source of KATRIN
}

\section{Sebastian FISCHER ${ }^{*}$}

Karlsruhe Institute of Technology

E-mail: sebastian.fischerakit.edu

The aim of the Karlsruhe Tritium Neutrino experiment (KATRIN) is the model-independent measurement of the neutrino mass by the investigation of the endpoint region of the tritium $\beta$ spectrum. For that purpose a windowless gaseous tritium source (WGTS) generates $\sim 10^{11} \beta$ electrons per second. The $\beta$-electrons are adiabatically guided to an electrostatic spectrometer which measures the integrated $\beta$-spectrum by varying the spectrometer voltage. In order to reach the design sensitivity of $200 \mathrm{meV} / \mathrm{c}^{2}$ (90\% C.L.) on the neutrino mass, the key parameters of the WGTS, i.e. column density, temperature, beta activity, and isotopic composition of the tritium gas have to be stabilised to the $0.1 \%$ level and accordingly monitored. An overview of the WGTS, its instrumentation and the current status will be given.

XV International Workshop on Neutrino Telescopes

March 11-15 2013

Venice, Italy

${ }^{*}$ Speaker. 


\section{Introduction}

The aim of the Karlsruhe Tritium Neutrino experiment (KATRIN) [1] is the model-independent measurement of the neutrino mass by the investigation of the endpoint region of the tritium $\beta$ spectrum. The design sensitivity of $200 \mathrm{meV} / \mathrm{c}^{2}$ (90\% C.L.) corresponds to an improvement by a factor of 10 in sensitivity on $m_{v}$ with respect to the upper limits obtained by the Mainz [2] and Troitsk [3,4] experiments and hence will allow for probing the cosmology relevant neutrino mass parameter space [5]. The experiment is currently being constructed at the Karlsruhe Institute of Technology by an international collaboration and will be operated for 5 years corresponding to 3 years of measurement time. An intense Windowless Gaseous Tritium Source (WGTS) will provide $10^{11} \beta$-electrons per second which are analysed by an electrostatic MAC-E filter [6, 7] with an energy resolution of $0.93 \mathrm{eV}$ at $18.6 \mathrm{keV}$ [1]. The key parameters of the WGTS, i.e. gas density, activity, temperature and isotopic composition of the source gas, have to be accurately monitored or, even better, stabilised to the $0.1 \%$ level as they are related to systematic uncertainties in the neutrino mass measurement: activity fluctuations of the WGTS, energy loss of $\beta$-electrons in the WGTS due to scattering, and the recoil and final state distribution of the daughter molecules [8]. In this work, an overview of the WGTS, its working principle, and the technical implementation will be given (section 2). Special focus will be laid on selected instrumentation for stabilisation and monitoring of the WGTS key parameters on the $0.1 \%$ scale (section 3 ).

\section{The KATRIN Windowless Gaseous Tritium Source (WGTS)}

The WGTS is a gas column created by continuously injecting tritium gas into the centre of a beam tube ( $10 \mathrm{~m}$ length, $9 \mathrm{~cm}$ diameter) and simultaneously pumping at both ends of the beam tube (figure 1). The beam tube is cooled to $30 \mathrm{~K}$ to reduce its conductance and the thermal motion of the gas molecules. By stabilising the gas injection rate, the beam tube temperature, and the pump rate to $0.1 \%$ each, a gas column with spatially inhomogeneous and temporally stable density profile is formed which acts as a $\beta$-electron source with low systematic uncertainties.

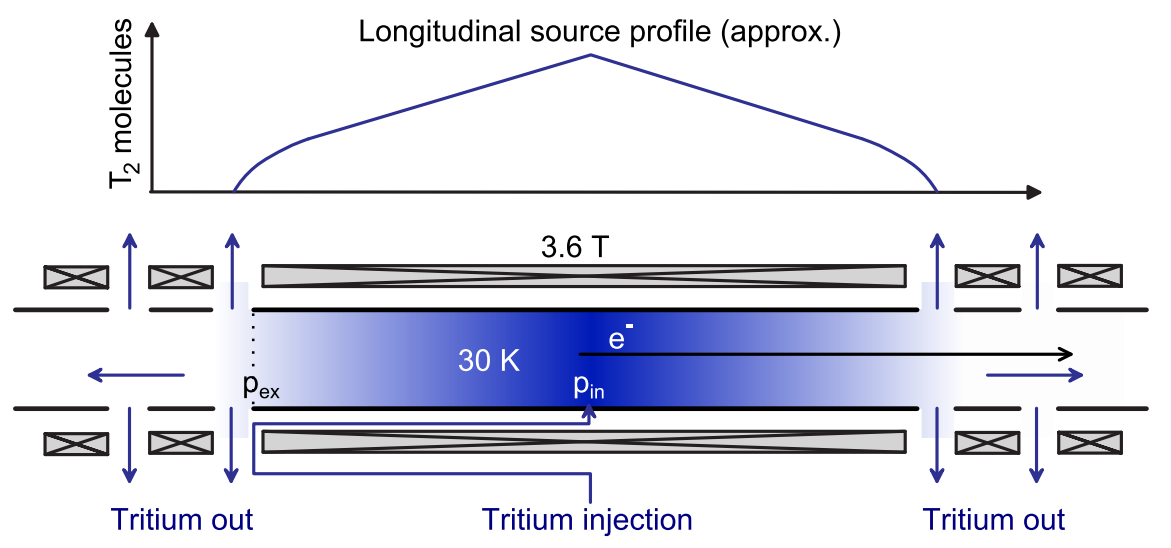

Figure 1: Schematic drawing of the windowless gaseous tritium source. The generated $\beta$-electrons are guided by magnetic field lines towards the spectrometer for energy analysis. 
(a)

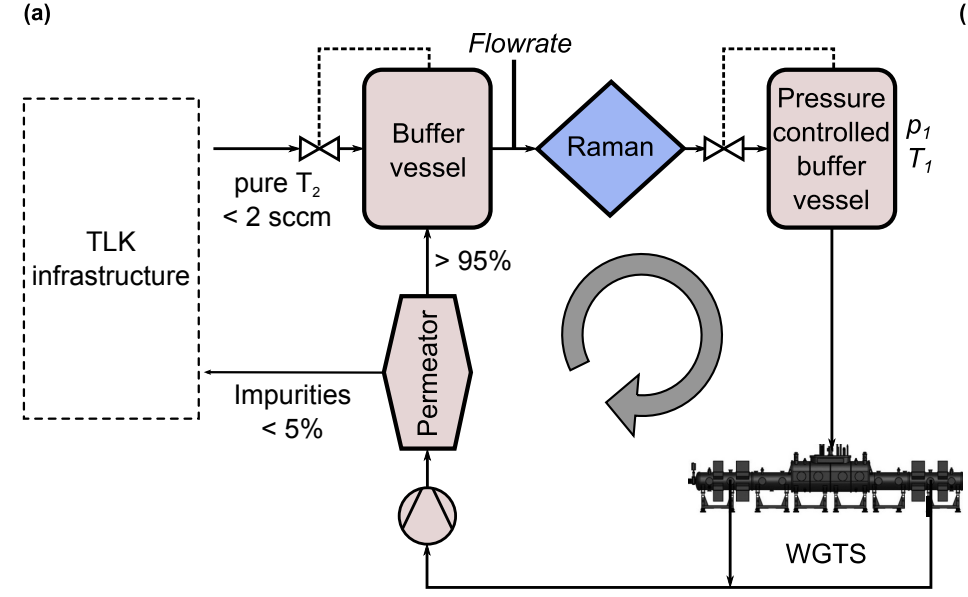

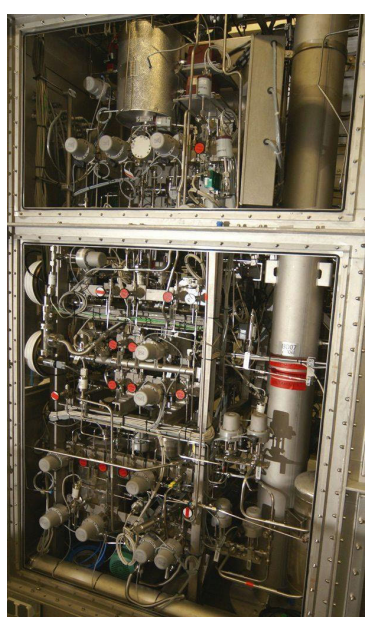

Figure 2: Simplified flow scheme (a) and picture (b) of the Inner Loop system. Only hydrogen isotopes can pass the permeator, that purifies the WGTS exhaust gas before being re-injected into the WGTS cryostat.

Three major systems are necessary for the operation of the WGTS: (i) The WGTS cryostat in which the beam tube, the cryo system for beam tube cooling and superconducting magnets for $\beta$-electron guidance are located, (ii) the Inner Loop system (figure 2) which is responsible for the stable tritium circulation through the WGTS cryostat, and (iii) the infrastructure of the Tritium Laboratory Karlsruhe (TLK) [9] for clean-up of the precipitated waste gases from the Inner Loop and supply of pure tritium.

\section{Control and monitoring of selected WGTS key parameters}

A multitude of systems are necessary to control and monitor the key parameters of the WGTS, i.e. column density, temperature, activity, and isotopic composition of the gas, to the $0.1 \%$ level. Selected systems will be briefly presented, while detailed information on all systems can be found in [8].

WGTS cryostat and beam tube temperature The beam tube temperature influences the column density, i.e. the number density of gas molecules in the WGTS, and hence has direct impact on the WGTS-activity but also on the scattering cross sections and energy loss of the $\beta$ electrons in the WGTS. Therefore a temperature stability of $0.1 \%$, i.e. $30 \mathrm{mK}$ at about $30 \mathrm{~K}$ is necessary. A novel cooling system utilizing two-phase neon has been developed for the WGTS cryostat $[10,11,12]$ and successfully tested in a pre-configuration of the WGTS in which only the beam tube and the cryogenic system was installed. A temperature stability of $1.5 \mathrm{mK} / \mathrm{h}$ was reached but the required temperature homogeneity along the beam tube axis not yet fully achieved [13]. Thorough investigations of the achieved temperature profiles lead to substantial optimization of the cryogenic design and a reduction of the thermal influence of the primary vacuum systems. Therefore the spatial temperature homogeneity is expected to be significantly improved in the final WGTS setup and to reach the required values of 
$\pm 30 \mathrm{mK}$ over its $10 \mathrm{~m}$ length. Currently the superconducting magnets and the tritium related components are being installed into the cryostat.

Inner Loop and tritium circulation The tritium gas is injected from a pressure controlled buffer vessel through a capillary with constant conductance into the WGTS beam tube (figure 2). The pumping rate at both ends of the beam tube is given by the rotation frequency of the turbo molecular pumps and typically stable to $10^{-4}$ [8]. The stability of the gas injection rate is hence determined by the pressure stability inside the buffer vessel. The palladium membrane filter ("Permeator" in figure 2) in the Inner Loop will separate the hydrogen isotopologues $\left(\mathrm{H}_{2}, \mathrm{HD}, \mathrm{D}_{2}, \mathrm{HT}, \mathrm{DT}, \mathrm{T}_{2}\right)$ from all other gas species that cannot permeate through the membrane. The Inner Loop was tested with nitrogen and deuterium to avoid tritium contamination of the system before connection with the WGTS cryostat. As placeholder for the WGTS beam tube a bypass tube with identical gas dynamical properties was used. The achieved pressure stability exceeded the $0.1 \%$ requirement by one order of magnitude, even under unrealistically large temperature cycling of the beam tube replacement, and thereby demonstrated the stable tritium circulation in the inner loop [8].

Gas composition The WGTS will be operated with tritium gas of $\geq 95 \%$ purity, that will typically consist of $\geq 90 \% \mathrm{~T}_{2}$ and $\leq 9 \%$ DT molecules, while other impurities (e.g. $\mathrm{D}_{2},{ }^{3} \mathrm{He}$, tritiated methane) are expected to be below $1 \%$ [1]. The knowledge of the gas composition is essential for the neutrino mass analysis since electron-gas scattering and the recoil and final states of the daughter molecules influence the $\beta$-spectrum differently depending on the gas molecule. The Raman spectroscopy system LARA continuously monitors the gas composition in the Inner Loop with $0.1 \%$ precision and about $3 \%$ accuracy $[14,15,16,17]$. Apart from the offline usage of Raman data for neutrino mass analysis, it will be also used online to operate the Inner loop: to decide when to feed pure tritium into the Inner Loop and to monitor the WGTS during commissioning with tritium when gas-wall and isotope exchange reactions [16] will be very prominent. Dedicated data analysis procedures have been developed $[18,19]$ and implemented into the data acquisition software such that Raman spectra are quantitatively evaluated within a second after acquisition and direct feedback is given to the Inner Loop operator. The LARA system is well tested and, apart from minor projected optimisations, ready for operation.

Source activity KATRIN will measure the integrated $\beta$-spectrum by subsequently stepping the retardation voltage of the MAC-E type spectrometer. The source activity has to be stable to $0.1 \%$ to avoid statistical variations between individual energy bins within a single $\beta$ spectrum and additionally to allow for the combination of data from different measurement runs. The WGTS source activity will be measured in-situ by Beta Induced X-ray Spectrometry (BIXS), i.e. by measuring the flux of X-Rays produced by $\beta$-electron impinging on a gold coated surface in the WGTS. The concept has been experimentally tested [20] and it was demonstrated that $0.2 \%$ changes can be detected already in a unoptimised prototype setup [21]. The KATRIN BIXS system is expected to reach a precision of $0.1 \%$ within about $50 \mathrm{~s}$ acquisition time, i.e. within a time interval similar to the Raman system [21]. The 
technical design of the BIXS-related vacuum system has been approved in autumn 2013 and most components of the BIXS system are already in stock at KIT.

\section{Conclusions}

The KATRIN Windowless Gaseous Tritium Source will set new standards in the field of ultra stable high luminosity tritium $\beta$ sources. Scientific requirements regarding long term stability, homogeneity and reliability have been met with unique new solutions in cryogenics, vacuum physics and measurement techniques. The WGTS cryostat is expected to be delivered to KIT in 2015 and thus will complete the KATRIN experiment.

\section{References}

[1] J. Angrik et al. (KATRIN collaboration), FZKA Scientific Report 7090 (2004) http://bibliothek.fzk.de/zb/berichte/FZKA7090.pdf.

[2] Ch. Kraus et al., Eur. Phys. J. C 40447 (2005) doi:10.1140/epjc/s2005-02139-7.

[3] V. M. Lobashev et al., Physics Letters B 460 227-235 (1999) doi:10.1140/epjc/s2005-02139-7.

[4] V. N. Aseev et al., Phys. Rev. D 84112003 (2011) doi:10.1103/PhysRevD.84.112003.

[5] The Planck Collaboration arXiv 1303.5076 [astro-ph.CO].

[6] P. Kruit and F. H. Read, J. Phys. E: Sci. Instrum. 16313 (1983) doi:10.1088/0022-3735/16/4/016.

[7] J. Beamson et al., J. Phys. E13 64 (1980) doi:10.1088/0022-3735/13/1/018.

[8] M. Babutzka et al., New Journal of Physics 14103046 (2012) doi:10.1088/1367-2630/14/10/103046.

[9] L. Dörr et al., Fusion Sci. Technol. 481262 (2005) http://www.new.ans.org/pubs/journals/fst/a_924.

[10] S. Grohmann et al., AIP Conf. Proc. 53B 1277-84 (2008) doi:10.1063/1.2908483.

[11] S. Grohmann, Cryogenics 49 413-420 (2009) doi:10.1016/j.cryogenics.2009.06.001.

[12] S. Grohmann et al., Cryogenics 51 438-445 (2011) doi:10.1016/j.cryogenics.2011.05.001.

[13] S. Grohmann et al., Cryogenics 55-56 5-11 (2013) 10.1016/j.cryogenics.2013.01.001.

[14] M. Schlösser et al., Fusion Sci. Technol. 603 976-981 (2011) http://www.new.ans.org/pubs/journals/fst/a_12579.

[15] M. Sturm, et al., Laser Physics 202 493-507 (2010) doi:10.1134/S1054660X10030163.

[16] S. Fischer et al., Fusion Sci. Technol. 603 925-930 (2011) http://www.new.ans.org/pubs/journals/fst/a_12567, arXiv 1208.1605v1 [physics.ins-det].

[17] M. Schlösser et al., J. Mol. Struct. 104461 (2013) doi:10.1016/j.molstruc.2012.11.022.

[18] T. M. James et al., Applied Spectroscopy $678949-959$ (2013) doi:10.1366/12-06766.

[19] M. Schlösser, B. Bornschein, S. Fischer, T. M. James, F. Kassel, S. Rupp, M. Sturm, H. H. Telle, Raman spectroscopy at the Tritium Laboratory Karlsruhe, submitted to Fusion Sci. Technol., (2013).

[20] M. Röllig et al., Fusion Eng. Des. 881263 (2013) doi:10.1016/j.fusengdes.2012.11.001.

[21] L. Bornschein, M. Sturm, B. Bornschein, M. Röllig and F. Priester, Status of the Neutrino Mass Experiment KATRIN, submitted to Fusion Sci. Technol., (2013). 